\title{
A prevenção de lesão por pressão em pacientes acompanhados pelo enfermeiro da estratégia de saúde da família
}

\author{
Pressure injury healing in patients followed up by nurses in the family health strategy \\ Prevención de lesiones por presión los pacientes acompañados por enfermeros de la \\ estrategia de la família
}

Daniele Fabíola Jaques ${ }^{1}$, Sabrina Ferreira Silva ${ }^{1 *}$, Andréia Andrade dos Santos ${ }^{1}$, Marcio Antonio Resende ${ }^{1}$, Renata Angélica Calsavara', Pâmella Aparecida Barros ${ }^{1}$, Gilberto de Souza1.

\section{RESUMO}

Objetivo: Revisar e identificar as medidas de prevenção e de promoção da saúde adotadas de modo a evitar o desenvolvimento da Lesão por Pressão em uma unidade de estratégia saúde da família. Revisão bibliográfica: Caracterizada pelo rompimento da integridade da pele, a LPP constitui hoje um grave problema de Saúde Pública, cuja incidência aumenta progressivamente em decorrência dos graus de dificuldade na terapêutica correta e principalmente da prevenção. As causas estão associadas a longos períodos de internação, uso exacerbado de medicamentos, déficit nutricional dentre outros fatores, tornando os clientes acamados os mais susceptíveis, devido rapidez e evolução das feridas cutâneas. As LPP constituem um importante problema vivenciado pelos profissionais da saúde, podendo ser consideradas um problema de saúde pública, pois além de gerar ônus, leva a transtornos físicos, mentais, emocionais o que acaba por influenciar nas taxas de morbimortalidade desses pacientes. Considerações Finais: Considerando a importância da atuação do enfermeiro da ESF, são vários desafios vivenciados pelo enfermeiro no tratamento das lesões por pressão, assim, cabe a esses profissionais manter-se atualizado nas estratégias voltadas para uma assistência integrada e eficiente dos pacientes com LPP.

Palavras-chave: Enfermagem, Estratégia saúde da família, Lesão por pressão.

\begin{abstract}
Objective: To review and identify health prevention and promotion measures adopted in order to avoid the development of Pressure Injury in a family health strategy unit. Literature review: Characterized by the disruption of skin integrity, $\mathrm{PI}$ is today a serious public health problem, whose incidence progressively increases due to the degrees of difficulty in correct therapy and especially Prevention. The causes are associated with long periods of hospitalization, exacerbated use of medications, nutritional deficit among other factors, making bedridden clients the most susceptible, due to speed and evolution of skin wounds. PI are an important problem experienced by health professionals, and can be considered a public health problem, because in addition to generating burden, leads to physical, mental, emotional disorders which ultimately influence stemming from the rates of morbidity and mortality of these patients. Final Considerations: Considering the importance of the nurses' actions, there are several challenges experienced by nurses in the treatment of pressure injuries, thus, it is up to these professionals to keep up to date in strategies aimed at a integrated and efficient care of patients with PI.
\end{abstract}

Key words: Nursing, Family health strategy, Pressure injury.

${ }^{1}$ Centro Universitário Presidente Tancredo de Almeida Neves (UNIPTAN), São João Del Rei - MG.

*E-mail: sabrina.11.15f@gmail.com

SUBMETIDO EM: 11/2019

ACEITO EM: 12/2019

PUBLICADO EM: 6/2020

REAS/EJCH | Vol.Sup.n.50 | e2313 | DOI: https://doi.org/10.25248/reas.e2313.2020 Página 1 de 7 


\section{RESUMEN}

Objetivo: Revisar e identificar las medidas de prevención y promoción de la salud adoptadas para evitar el desarrollo de lesiones a presión en una unidad de estrategia de salud familiar. Revisión de la literatura: Caracterizada por la alteración de la integridad de la piel, LPP es hoy un grave problema de salud pública, cuya incidencia aumenta progresivamente debido a los grados de dificultad en la terapia correcta y especialmente Prevención. Las causas están asociadas con largos períodos de hospitalización, uso exacerbado de medicamentos, déficit nutricional entre otros factores, haciendo que los clientes postrados en cama sean los más susceptibles, debido a la velocidad y evolución de las heridas de la piel. Los LPP son un problema importante experimentado por los profesionales de la salud, y pueden considerarse un problema de salud pública, porque además de generar carga, conduce a trastornos físicos, mentales, emocionales que en última instancia influyen morbilidad y mortalidad de estos pacientes. Consideraciones finales: Teniendo en cuenta la importancia de las acciones de los enfermeros fhs, hay varios desafíos experimentados por las enfermeras en el tratamiento de lesiones por presión, por lo tanto, depende de estos profesionales mantenerse al día en estrategias dirigidas a una atención integrada y eficiente de los pacientes con LPP.

Palabras clave: Enfermería, Estrategia de salud familiar, Lesión por presión.

\section{INTRODUÇÃO}

As lesões por pressão (LPP) são caracterizada pelo rompimento da integridade da pele, decorrente de inúmeros fatores dentre os quais citaremos no decorrer do seu desenvolvimento, bem como dos processos atualizados de reparação dos tecidos. Frequentemente os pacientes com esse tipo de lesão são cuidados pelo Sistema Único de Saúde (SUS) (ALBUQUERQUE AM, et al., 2018).

As LPP constituem um importante problema vivenciado pelos profissionais da saúde, podendo ser consideradas um problema de saúde pública, pois além de gerar ônus, leva a transtornos físicos, mentais, emocionais o que acaba por influenciar nas taxas de morbimortalidade desses pacientes, seja no domicílio ou nas instituições hospitalares e, em particular, nas unidades de terapia intensiva (UTI), visto que os pacientes encontram-se acamados, geralmente sem condições de mobilização em decorrência da gravidade do seu quadro clínico (ALBUQUERQUE AM, et al., 2018).

Há um aumento da prevalência de LPP nos últimos anos convenientes à maior expectativa de vida da população consecutivo de avanços na assistência à saúde, onde possibilitou a sobrevida dos pacientes com doenças graves e previamente letais, transformadas em doenças crônicas e vagarosamente debilitantes (WADA A, et al., 2010). A avaliação completa do paciente implica em ações voltadas para o enfermeiro, este, que deverá estar prontamente preparado e apto para tratar e curar as lesões cutâneas, contando com conhecimentos embasados na melhoria da terapêutica a ser oferecida e prestada ao paciente.

Como o profissional de enfermagem está diretamente ligado ao tratamento de feridas, nos três níveis hierárquicos de saúde, deve resgatar a responsabilidade de manter a observação intensiva relacionada ao local, via sanguínea e via externa relacionados ao favorecimento da lesão ou interfiram no processo de cicatrização, assim como ser o responsável pela referência e contra referência do atendimento e acompanhar a total recuperação e reabilitação do paciente (MORAES JT, et al., 2016).

Torna-se fundamental e imprescindível que o profissional enfermeiro aperfeiçoe e conheça os tipos de curativos e seu mecanismo de ação na ferida a ser tratada, para que perceba o momento certo de se aplicar à técnica ou o tipo de curativo requerido naquele momento (MORAES JT, et al., 2016).

O enfermeiro da Estratégia Saúde da Família (ESF), nível primário de atenção do SUS, empenha a promoção da saúde como perspectiva de articulação transversal, o que concebe visibilidade as condições que colocam em risco a saúde da população e as diferenças entre cultura, território e necessidades presentes no Brasil, tendo em vista a criação de meios que reduzam a fragilidade e incorporem o controle e participação social na gestão das políticas públicas de saúde (MOTTA LCS e SIQUEIRA-BATISTA R, 2018). 
Com o intuito de promover a qualidade de vida do paciente, é preciso, além da cicatrização e do fechamento das lesões, de um processo de tomada de decisão quanto aos procedimentos, recursos e tecnologias que serão utilizados. Reitera-se a necessidade do desenvolvimento de estratégias educativas para qualificação dos profissionais, uma vez que a falta de conhecimento impacta diretamente sobre as ações preventivas e, consequentemente, sobre a incidência e prevalência da LPP (ALBUQUERQUE AM, et al., 2018).

Um planejamento de cuidados preciso, uma avaliação satisfatória, que possa prevenir a LPP, assim como a promoção da saúde, com o envolvimento do indivíduo, da família e população adscrita, tornam-se possíveis alternativas frente aos desafios, na criação de um novo atendimento no contexto da Atenção Primária da Saúde (SOARES CF e HEIDEMANN ITSB, 2018). Dessa forma o objetivo desse estudo é revisar e identificar as medidas de prevenção e de promoção da saúde adotadas, de modo a evitar o desenvolvimento da Lesão por Pressão em uma unidade de estratégia saúde da família.

\section{REVISÃO BIBLIOGRÁFICA}

\section{As Lesões por pressão e suas implicações}

Caracterizada como um indicador negativo de qualidade do cuidado, as LPP são causadas pelo comprometimento da pele devido rompimento de sua integridade, desencadeada por uma ferida cutânea e representa atualmente um grande desafio para o cuidado em saúde, o que compromete a qualidade de vida do cidadão e leva a complicações como mortalidade, além dos custos onerosos e pelo tempo gasto para sua recuperação (BERNARDES RM e CALIRI MHL, 2016). A terminologia "úlcera por pressão" foi modificada para "lesão por pressão", pois a palavra "lesão" apresenta mais precisão a destruição tecidual em pele ulcerada e/ou intacta (NPUAP, 2016).

Lesão por pressão é um dano localizado na pele e/ou nos tecidos moles subjacentes, geralmente em áreas ósseas ou pelo paciente estar usando algum dispositivo por indicação médica de outro artefato. Podendo apresentar ferida sem prejuízos na pele ou exposta apresentando ou não sintomas como dor, odor fétido ou secreção. Sendo resultante de exposição por muito tempo a umidade, pressão local, ausência de mudança de decúbito, pelo microclima, nutrição, perfusão, comorbidades e pela sua condição (PUAP, 2014).

Estudos apresentaram que no Brasil, entre $41,2 \%$ e $59 \%$ de risco para o avanço da LPP, e uma prevalência entre $8 \%$ e $23 \%$, tornando-se um fator muito sério por se tratar de um evento adverso que em apenas $5 \%$ dos casos não se pode prevenir, de acordo com a Declaração do Rio de Janeiro sobre a Prevenção da LPP (BORGES EL e FERNANDES FP, 2014). Diante do crescente número de pessoas

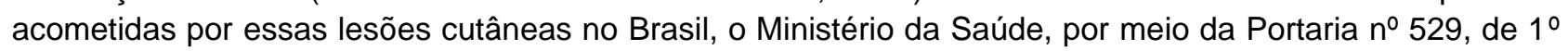
de abril de 2013, instituiu o Programa Nacional de Segurança do Paciente, no qual um dos objetivos é a diminuição da ocorrência da LPP (BRASIL, 2013).

O National Pressure Ulcer Advisory Panel (NPUAP) fortaleceu o conceito e adicionou novas apresentações da LPP como lesões por pressão não Estadiável: Destruição total da pele em sua consistência e destruição tissular que não está visível pois encontra-se encoberta por escara ou esfacelo; e Lesão por Pressão Tissular Profunda: Coloração avermelhado com tonalidades marrom ou púrpura, persistente e que não alveja; Lesão por Pressão por causa de uso de dispositivos de indicação médica e Lesão por Pressão em Membrana Mucosa (MORAES JT, et al., 2016).

Refere-se a uma organização profissional independente sem fins econômicos empenhados ao gerenciamento e a prevenção de LPP. O NPUAP surgiu em 1986, formado por especialistas qualificados em cuidados à saúde de diversas disciplinas. Serve como instrução para o governo, profissionais da saúde, o público e agências de cuidados de saúde, sendo, portanto, uma entidade reconhecida internacionalmente (NPUAP, 2016).

As lesões por pressão geralmente são ocasionadas por diversos fatores, externos ou internos, por traumas físicos, químicos, agentes mecânicos dos mais diversificados, bem como por afecção clínica, a 
maioria delas por períodos prolongados de internação, em que diversos fatores sistêmicos ficam comprometidos influenciando negativamente no processo de cicatrização (BRITO TB, 2017). Alterações nutricionais, deficientes ou alimentação inadequada, associada à baixa imunidade, uso excessivo de medicamentos, principalmente imunossupressores, bem como doenças crônicas como insuficiências circulatórias e a diabetes, associadas ou não ao etilismo e ao tabagismo caracterizam a deficiência no processo de cura (MORAES JT, et al., 2016).

Quanto à localização das úlceras, os achados corroboram estudos nacionais e internacionais há predomínio das úlceras na região sacral, trocanteriana e calcânea, consideradas locais de apoio quando o paciente está em decúbito dorsal ou lateral (comuns entre pacientes críticos) (MORAES et al, 2018, p. 265).

A prevenção da LPP constitui ação primordial, visto que tal agravo causa dor e desconforto para 0 paciente e família, podendo retardar sua recuperação. $O$ tratamento gera custos à instituição e aumento na demanda de trabalho da enfermagem (MIYAZAKI MY, et al., 2010). A ocorrência de LPP é considerada um evento adverso que pode, mas na maior parte das vezes são evitadas, existe algumas ocasiões que mesmo utilizando medidas de prevenção, as condições fisiológicas do paciente tornam as lesões inevitáveis (PALAGI S, et al., 2015).

De acordo com a gravidade e intensidade das lesões, estas podem ser classificadas em quatro estágios. O Estágio I: Pele Íntegra com eritema não-branqueável; Estágio II: Perda de espessura parcial da pele com exposição da derme; Estágio III: perda total da espessura da pele e por último Estágio IV: perda total da espessura da pele e perda tissular, podendo haver até um Estágio $\mathrm{V}$ em algumas literaturas: perda da integridade da pele com perda tissular não visível (NPUAP, 2014).

O enfermeiro é o profissional responsável por avaliar a lesão com a finalidade de promover a cicatrização, eliminando fatores que possam retardá-la, tratar e prevenir infecções, prevenir contaminação exógena, proteger a ferida contra traumas, manter a umidade da ferida e limpá-la. Sistematizando o processo de avaliação da ferida para escolha de melhor tratamento e auxiliando o organismo a promover a cicatrização da lesão (SANTOS SH, et al., 2008).

O profissional deve classificar a lesão quanto ao tempo de evolução: aguda ou crônica; Quanto ao estado microbiológico: contaminada, colonizada e infectada; Quanto ao comprometimento das camadas da pele e estruturas adjacentes: superfície e profunda; Quanto à localização descrição anatômica; Quanto ao exsudato e aspecto: (seroso, serosanguinolento, sanguinolento, piosanguinolento, purulento). Cor: amarelado, esbranquiçado, esverdeado, cinza, acastanhado. Odor: característico, fétido, pútrido (SANTOS $\mathrm{SH}$, et al., 2008).

Por se tratar de lesões causadas por longos períodos de internação, pacientes hospitalizados e acamados, se faz imprescindível acompanhamento rotineiro das lesões cutâneas para devida avaliação contínua do tratamento associado a uma série de intervenções de enfermagem prescritos na grande maioria das vezes pelo enfermeiro (a) do ESF.

\section{A importância da ESF no tratamento das LPP}

Implantado como modelo de atenção à saúde no Brasil, o SUS, através da lei 8080/90 foi marcado pela assistência médica, individualista e curativa, que reafirma o direito à saúde e a diminuição da diferença dos direitos assegurados por lei e as ações e serviços de saúde ofertados a todos como dever do Estado (LIMA EFA, et al., 2016).

Com o seu surgimento em 1988 na Constituição Federal, o SUS fortaleceu a Atenção Primária em Saúde (representando o primeiro lugar de atenção à saúde, mantendo a integralidade como princípio e como ações, a prevenção, promoção, tratamento e recuperação da saúde). Além disso, o SUS é organizado simultaneamente por cinco princípios: regionalização, hierarquização, resolutividade, descentralização, participação social e a complementariedade (TOSIN C, et al., 2017).

Tendo como a essência do cuidado às pessoas com lesões por pressão, tem-se que tais ações tanto curativas quanto preventivas devem ser estabelecidas em média e alta complexidade, assim como na 
Atenção Primária à Saúde (APS), pela ESF, que estão cada vez mais empenhados para o estabelecimento de diretrizes que conduzem a prática da prevenção, e consequentemente promovendo a qualidade de vida ao indivíduo. (SOARES CF e HEIDEMANN ITSB, 2018).

Com intuito de fortalecer a APS e suas práticas assistenciais, criou-se em 1994, a Estratégia Saúde da Família (ESF), inicialmente introduzido como PSF (Programa de Saúde da Família), sendo posteriormente modificado, aprimorado em 2006 passou a ser estratégia, melhorando o alcance e acesso dos indivíduos aos serviços de saúde (FERTONANI HP, et al., 2015).

Composta por uma equipe mínima tem-se no ESF, médico, enfermeiro, auxiliar de enfermagem ou técnico em enfermagem e agentes comunitários de saúde capacitados e encarregados de proporcionar assistência a uma população adscrita, estabelecida em uma determinada área geográfica, por meio de práticas nas unidades de saúde, nas comunidades e nos domicílios, podendo acrescentar os profissionais de saúde bucal, de acordo com a necessidade do município (LIMA EFA, et al., 2016).

Um atendimento adequado, um plano de cuidados bem estabelecido para prevenir a ferida, assim como atividades que visam melhorar a saúde, com o envolvimento da pessoa, da família e da comunidade, mostram-se como opções frente aos problemas (SOARES CF e HEIDEMANN ITSB, 2018). No intuito de minimizar complicações e reduzir o risco de agravos, o profissional enfermeiro como responsável integrante da ESF passa a exercer sua função assistindo da melhor forma o paciente, com intuito de proporcionar ao cliente e familiares o processo de autocuidado (SOARES CF e HEIDEMANN ITSB, 2018).

\section{O papel do enfermeiro da ESF nas condutas preventivas: cuidado com as LPP}

O enfermeiro é o responsável pelo cuidado. Sendo assim deve focar na prevenção de agravos, promover saúde, norteando a prática assistencial, busacando um número cada vez menor de lesão por pressão (SOARES CF e HEIDEMANN ITSB, 2018). O tratamento das LPP implica em conhecimentos, que vão desde o entendimento sobre anatomia e fisiologia da pele até o domínio de técnicas e produtos atualmente colocados no mercado. A avaliação das condições de saúde do paciente, sua história pregressa, seus hábitos alimentares e suas condições de moradia e saneamento são pontos importantes no processo de cura de um paciente com LPP ou qualquer outro tipo de ferida cutânea (SOARES CF e HEIDEMANN ITSB, 2018).

O cuidado com lesões é realizado principalmente pela equipe de enfermagem, sendo o enfermeiro líder de equipe, porém não é exclusivo desta área profissional, uma vez que o cuidado de feridas deve ser implementado em uma visão interdisciplinar (MORAES JT, et al., 2016). Os enfermeiros que prestam essa assistência devem dominar o conteúdo e saber avaliar as lesões e adotar medidas de aferição e análise periódica das condições da lesão e do paciente em conjunto, de maneira holística, comtemplando aspectos inerentes á idade, doenças crônicas, condições nutricionais, repouso, uso de medicamentos, entre outros, fatores estes que contribuem para o avanço ou retardo da cicatrização (MORAES JT, et al., 2016).

Deverá o enfermeiro, buscar a preservação das potencialidades e bem-estar das pessoas que os utilizam. Para isso é fundamental que haja participação, envolvimento e respeito às opiniões do paciente e da família. Sabe-se que o profissional de enfermagem possui um papel fundamental no que se refere ao cuidado integral ao paciente, bem como desempenhar um trabalho de alta relevância no tratamento de feridas, uma vez que tem maior contato com o mesmo, acompanha o processo evolutivo da lesão, orienta, executa e supervisiona o curativo, detêm maior domínio desta técnica, em virtude da sua capacitação profissional respaldo para atuar em equipe com outros profissionais junto a enfermagem desenvolvê-la como uma de suas atribuições (MORAES JT, et al., 2016).

É de total responsabilidade do enfermeiro, avaliar todas as condições que envolvem o tratamento das lesões devendo este, obter conhecimento de protocolos institucionais, realizar treinamentos especializados, entender de mensuração de feridas e de todas as modalidades de tratamento de LPP. É preciso que existam condições materiais e aprimoramento em relação a todos os aspectos que envolvam a cura da ferida. Outro aspecto fundamental na prevenção da LPP é a capacitação dos profissionais de enfermagem. Um estudo, que analisou o conhecimento sobre a prevenção de lesão por pressão da equipe de 
enfermagem que presta assistência direta ao paciente adulto e idoso em um hospital universitário de Minas Gerais, constatou que tanto o enfermeiro quanto o técnico de enfermagem apresentaram desempenho menor que o esperado (MIYAZAKI MY, et al., 2010).

Avaliar o conhecimento dos profissionais é essencial para implementar ações educativas em vários contextos de atendimento para cuidar adequadamente de pessoas evitando alta incidência de erros nas medidas preventivas direcionadas (FIGUEIREDO ZM, et al., 2010). O profissional valorizado no mercado de trabalho é aquele com formação cientifica e pensamento crítico capaz de solucionar problemas de forma intregral e da melhor maneira possível, pensando nas deficiências de matérias e estrutura física da unidade (FURUKAWA PO e CUNHA ICKO, 2010).

Para evitar úlceras, além do tratamento medicamento, ao profissional enfermeiro deve avaliar sistematicamente com dispositivos como a escala de Braden para prevenir novas lesões, além da prescrição de medidas não farmacológicas (BORGES EL e FERNANDES FP, 2014). O uso desta escala é de grande importância na prevenção aumentando a qualidade do serviço, para isso é necessário a capacitação recorrente do enfermeiro e a busca por novos conhecimentos (BERNARDES RM e CALIRI MHL, 2014).

No entanto, é indispensável um olhar clínico do enfermeiro, como também vários aspectos importantes, por exemplo, o controle de diabetes mellitus e hipertensão, avaliações nutricionais, medicamentosos, infecciosos e, principalmente, um cuidado educativo extremamente qualificado (MORAES JT, et al., 2016). Diante de um mercado que dispõe de produtos cada vez mais diversificados, cabe ao enfermeiro estar buscando atualizações com relação a conhecimentos e pesquisas diversas direcionadas ao tratamento da LPP, para que cada dia mais possa estar aprimorando suas técnicas e utilizando cada vez melhor o produto e a técnica adequada para cada fase do processo de cicatrização, associando prescrições específicas e valiosas na conquista pelo sucesso (BORGES EL e FERNANDES FP, 2014).

Segundo Moraes JT, et al. (2016), A uma grande importância significativa na associação dos curativos que serão aplicados a partir do método de tratamento de acordo com a evolução da ferida e seus aspectos. Dessa forma, a atualização e o embasamento científico auxiliará o enfermeiro na escolha do tratamento correto e servirá de subsídio para elaboração e desenvolvimento de uma assistência individual e adequada conforme o quadro clinico do paciente de cuidados com métodos favoráveis para executá-lo, possibilitando ao paciente conforto e uma cicatrização eficaz (MORAES JT, et al., 2016).

Consideram-se ainda critérios preventivos de LPP como de cura, o avanço da idade, o metabolismo diminuído, caracterizando uma fase de deficiências de substâncias responsáveis pela proteção do organismo, associado às doenças associadas, dentre as mais comumente encontradas (hipertensão e diabetes), são fundamentais neste processo, pois para se obter bons resultados é imprescindível controlar primeiramente as patologias de base e ainda, o controle nutricional que favorece no processo de cicatrização (BORGES EL e FERNANDES FP, 2014).

Toda prevenção exige do profissional enfermeiro a capacidade de observar mínimos detalhes diariamente em relação à ferida e ao paciente, cabendo-lhe a tarefa de adquirir muito conhecimento e bom senso na descoberta da técnica mais indicada entre tantas disponíveis no mercado e promover uma melhor qualidade de vida para o paciente.

\section{CONSIDERAÇÕES FINAIS}

Considerando a importância da atuação do enfermeiro da ESF, vários são os desafios vivenciados por este profissional no tratamento das LPP. Devido à maneira com que as Unidades de saúde são organizadas, podem ainda não oferecer condições para inserir programas apropriados e investimento correto da terapêutica ideal para cada tipo de lesão, sendo a avaliação diária é essencial. A capacitação e busca por conhecimentos do profissional enfermeiro, para melhorias nas técnicas e coberturas das mais inovadoras para se obter resultados excepcionais é de suma importância. Contudo, compete aos profissionais manter-se atualizado nas estratégias voltadas para uma assistência integrada e eficiente dos pacientes com LPP, principalmente dos acamados, que demandam maiores cuidados para que obtenha êxito na prevenção das LPP. 


\section{REFERÊNCIAS}

1. ALBUQUERQUE AM, et al. Este de conhecimento sobre lesão por pressão. Revista Enfermagem UFPE. 2018.

2. BERNARDES RM e CALIRI MHL. Pressure ulcer prevalence in emergency hospitals: a cross-sectional study. Online Braz J Nurs 2016.

3. BRASIL. Ministério da Saúde (BR). Portaria № 529, de 1ำ de abril de 2013. Institui o Programa Nacional de Segurança do Paciente (PNSP). Brasília (DF): Ministério da Saúde; 2013.

4. BRITO TB. Fatores de risco e incidência de lesão por pressão em pacientes internados em uma Unidade de Terapia Intensiva do Estado de Roraima. Boa Vista, RR. 2017, Monografia apresentada à Universidade Federal de Roraima.

5. BORGES EL e FERNANDES FP. Úlcera por pressão. Manual para prevenção de lesões de pele: recomendações baseadas em evidências. 2014.

6. FERREIRA AM, et al. The use of fatty acids in wound care: an integrative review of the Brazilian literature. Rev Esc Enferm USP. 2012.

7. FERTONANI HP, et al. Modelo assistencial em saúde: conceitos e desafios para a atenção básica brasileira. Ciência \& Saúde Coletiva. 2015.

8. FIGUEIREDO ZM, et al. Pressure Ulcers in Patients with Spinal Cord Injury: Knowledge of Relatives and Caregivers. Av. Enferm. 2010.

9. FURUKAWA PO e CUNHA ICKO. From management competencies to nurse managerial competencies. Rev Bras Enferm. 2010.

10. LIMA EFA, et al. Avaliação da estratégia saúde da família na perspectiva dos profissionais de saúde. Escola Anna Nery Revista de Enfermagem, 2016.

11. MIYAZAKI MY, et al. Conhecimento dos profissionais de enfermagem sobre prevenção da úlcera por pressão. Rev Latino-Am Enfermagem. 2010.

12. MORAES JT, et al. Conceito e classificação de lesão por pressão: atualização do National Pressure Ulcer Advisory Panel. Revista Enferm. Cent. O. Min. 2016.

13. MOTTA LCS e SIQUEIRA-BATISTA R. Estratégia Saúde da Família: Clínica e Crítica. Revista Brasileira de Educação Médica. 2015.

14. NATIONAL PRESSURE ULCER ADVISORY PANEL (NPUAP) European Pressure Ulcer Advisory Panel, Pan Pacific Pressure Injury Aliance. Prevention and Treatment of Pressure Ulcers: Clinical Practice Guideline. Washington: NPUAP/EPAUAP/PPPIA; 2014.

15. NATIONAL PRESSURE ULCER ADVISORY PANEL (NPUAP); European Pressure Ulcer Advisory Panel and Pan Pacific Pressure Injury Alliance. Prevention and treatment of pressure ulcers: quick reference guide. Emely Haersler (Ed.). Combrige Media: Osborne Park, Austrália; 2014.

16. NATIONAL PRESSURE ULCER ADVISORY PANEL (NPUAP). Pressure Ulcer Stages Revised. Washington, 2016.

17. NATIONAL PRESSURE ULCER ADVISORY PANEL (NPUAP) announces a change in terminology from pressure ulcer to pressure injury and updates the stages of pressure injury. NPUAP News 2016.

18. PALAGI S, et al. Laser therapy in pressure ulcers: evaluation by the Pressure Ulcer Scale for Healing and Nursing Outcomes Classification. Rev Esc Enferm USP. 2015.

19. SANTOS SH, et al. Avaliação de feridas pelos enfermeiros de instituições da rede pública. 2008.

20. SCHNEIDER DG e RAMOS FRS. Processos éticos de enfermagem no Estado de Santa Catarina: caracterização de elementos fáticos. Rev. Latino-Am. Enfermagem. 2012.

21. SOARES CF e HEIDEMANN ITSB. Promoção da saúde e prevenção da lesão por pressão: expectativas do enfermeiro da Atenção Primária. Texto Contexto Enferm.; 2018.

22. TOSIN C, et al. A transição do modelo tradicional para estratégia saúde da família: a percepção do usuário. Cadernos da Escola de Saúde. 2017.

23. WADA A, et al. Úlceras por pressão. Rev. med. Hosp. Univ. 2010. 